\title{
ALK-positive pulmonary adenocarcinoma with signet ring features (PASRF) and polygonal cell morphology simultaneously co-expressing TTF-1/p63/P40: a case report
}

\author{
Yang Tan ${ }^{1 \#}$, Xiaofang $\mathrm{Lu}^{1 \#}$, Yun $\mathrm{Li}^{2}$, Dingzhun Liao ${ }^{1}$, Wenlin Xie ${ }^{1}$, Jian Song ${ }^{1}$, Ling Xue ${ }^{1}$, Junfeng Zhu ${ }^{1}$ \\ ${ }^{1}$ Department of Pathology, The Seventh Affiliated Hospital of Sun Yat-sen University, Shenzhen, China; ${ }^{2}$ Department of Thoracic Surgery, The \\ Seventh Affiliated Hospital of Sun Yat-sen University, Shenzhen, China \\ \#These authors contributed equally to this work. \\ Correspondence to: Ling Xue, MD; Junfeng Zhu, MD. Department of Pathology, The Seventh Affiliated Hospital of Sun Yat-sen University, Shenzhen, \\ China. Email: xuel@mail.sysu.edu.cn; zjf20102@163.com.
}

\begin{abstract}
Pulmonary adenocarcinoma with signet ring features (PASRF) is relatively rare and often harbors anaplastic large cell kinase gene (ALK) rearrangements. As patients with ALK+ lung adenocarcinoma typically present at advanced stages, it is important to detect ALK+ PASRF at metastatic sites to ensure ALK-targeted therapy can be best applied. In this study, we report a case of PASRF with polygonal cell morphology in an 85-year-old female who had metastasized to the supraclavicular lymph node and was treated with Alectinib, a novel small molecule tyrosine kinase inhibitor targeting ALK. Biopsy of the node revealed numerous microscopic neoplastic cells which had formed nests or cords, in addition to signet ring cells (60\%), and another morphology of polygonal cells (40\%) which contained eosinophilic cytoplasm. Mitotic images were common, and necrosis was observed. Immunohistochemically, both morphology tumor cells were diffusely positive for TTF1, CK7, and Napsin A, and most of the two were positive for P40, P63, and CK5/6 and negative for CK20 and CDX2. Co-expression of TTF-1, CK7, Napsin A, and P40, P63, and CK5/6 were overtly present, and Alcian blue-periodic acid-Schiff (AB-PAS) staining showed intracytoplasmic mucin in the two cells. In addition, ALK rearrangements were detected by ALK (clone D5F3) immunohistochemical assay and fluorescence in situ hybridization (FISH), A final diagnosis of metastatic ALK-positive PASRF with polygonal cell morphology to the supraclavicular lymph node was made, and the patient was treated with Alectinib, which brought a reduction in tumor size and good results.
\end{abstract}

Keywords: Pulmonary adenocarcinoma; signet ring cell; polygonal cell; lymph node metastasis; case report

Submitted Feb 23, 2021. Accepted for publication May 14, 2021.

doi: $10.21037 /$ tcr-21-335

View this article at: https://dx.doi.org/10.21037/tcr-21-335

\section{Introduction}

PASRF was considered a rare, distinct subtype of pulmonary adenocarcinoma and designated as signet ring adenocarcinoma in the 2004 WHO classification of lung tumors (1). However, in 2015 WHO classification of lung tumors, PASRF was removed from the variant because the new classification was based on tumor growth patterns, not tumor cell morphology. Signet ring cell morphology is now viewed as a cytologic change that may occur in association with various histological patterns rather than as a separate subtype (2). PASRF is usually found with classic adenocarcinoma (3), and the most common histological growth pattern associated with the tumor is the solid subtype, with which its poor prognosis is associated (3). Less commonly, PASRF is found with acinar and papillary subtypes, in which the amount of signet ring cells varies among cases (4). PASRF are also commonly associated with a specific molecular alteration involving ALK gene rearrangement with the formation of the ALK-EML4 fusion gene and benefit from ALK tyrosine-kinase inhibitor 

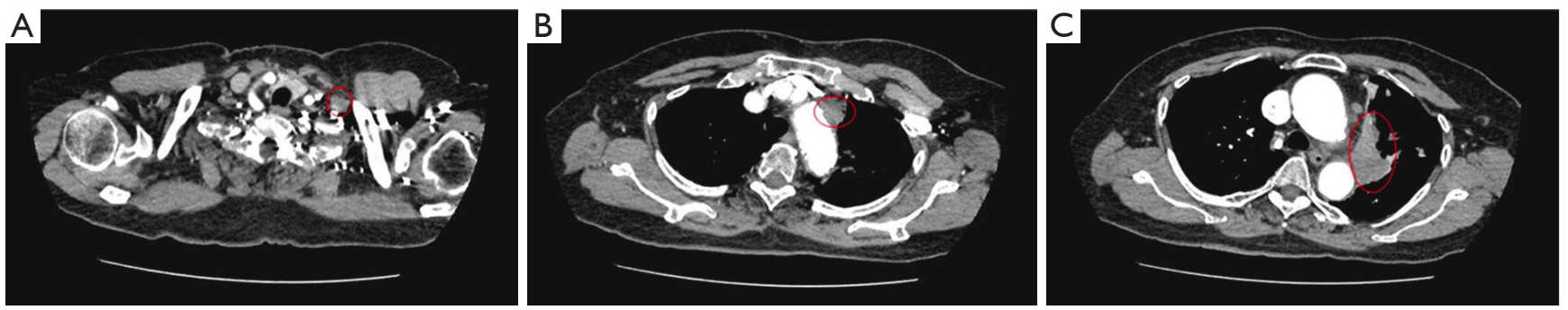

Figure 1 Computed tomography (CT) of the chest at the time of diagnosis on August 20, 2019. The red circle indicates the supraclavicular lymph node (A), mediastinal lymph nodes (B), and the lung tumor (C).

therapies (5-8). PASRF cases frequently present at advanced stages after having metastasized to lymph nodes $(1,2,4,9)$, and stage IV disease is found in $6.5-16.2 \%$ of patients (10). A case of leptomeningeal carcinomatosis has even been reported (11). Therefore, recognition of PASRF is of great clinical significance, especially at metastasis sites.

In this study, we describe a case of ALK-positive PASRF with polygonal cell morphology metastatic to the supraclavicular lymph node and discuss the differential diagnostic considerations. Recognition of this rare morphology is of great importance for the correct diagnosis of PASRF and patients undergoing ALK-targeted therapy. We present the following article in accordance with the CARE reporting checklist (available at https://dx.doi. org/10.21037/tcr-21-335).

\section{Case presentation}

An 85-year-old Chinese Han female presented to the Guangming District Central Hospital on August 13, 2019, complaining of general fatigue. Examinations, including chest computed tomography (CT), suggested the possibility of cancer of the central left lung. The patient was further consulted at the Department of Oncology of the 7th Affiliated Hospital of Sun Yat-Sen University on August 17, 2019 , and was found to have a mild, dry cough but reported no chest tightness, shortness of breath, fever, nausea, or vomiting. While defecation and urination were reported as normal, with no significant change in body weight, physical examination revealed an enlarged palpable lymph node of $1.7 \times 1.5 \mathrm{~cm}$ in size in the left supraclavicular region with moderate mobility. Other superficial lymph nodes were not palpable. The patient had a history of hypertension and hypokalemia for 18 years but was not a smoker and reported no family history of lung cancer.

Both plain and enhanced CT scans were performed on
August 20, 2019 (Figure 1A,B,C) and showed an irregular tumor beside the hilum of the upper left lung, with a lobulated margin and a size of about $45 \times 38 \times 57 \mathrm{~mm}$. The medial part was close to the mediastinal pleura, and irregular thickening of the local pleura was observed. The lesion resulted in the truncation of the upper left lung bronchus, and low-density lesion filling was observed in some bronchus. After contrast enhancement, the tumor showed mild-to-moderate inhomogeneous enhancement, and the adjacent vessels were surrounded by partial stenosis. The left peripheral lung anterior and lingual segments showed strip-like consolidation and hardening, which were significantly enhanced after contrast, and the left upper lobe lymphoid stroma was thickened, with grid-like, nodular, and ground-glass density areas. Multiple enlarged lymph nodes were found in the mediastinum, left lung hilum, and left the supraclavicular region, of which the largest was about $23 \times 16 \mathrm{~mm}$.

The results of tumor markers (chemiluminescent immunoassay) were as follows: CEA $18.04 \mathrm{ng} / \mathrm{mL}$ (normal: 0-5.00), CA125 267.15 U/mL (normal: 0-35.00), CA15-3 49.26 U/mL (normal: 0-35.00), AFP $3.05 \mathrm{ng} / \mathrm{mL}$ (normal: 2-20.00), and CA19-9 29.24 U/mL (normal: 0-35.00). The clinical diagnosis was lung cancer in the upper lobe of the left lung with peripheral obstructive inflammation, atelectasis with carcinomatous lymphangitis and intrapulmonary metastasis, invasion of the mediastinal pleura, and lymph node metastases in the mediastinum left hilum and left supraclavicular areas.

Percutaneous needle core biopsy of the left supraclavicular lymph node was carried out to identify the pathological diagnosis and determine treatment. On August 22, 2019, ultrasound-guided left supraclavicular lymph node biopsy under local anesthesia was performed by using an 18-G biopsy needle, and three gray-white tissues were obtained. Microscopically, tightly packed nests of tumor cells infiltrated the fibrous stroma tissue, and focal necrosis 

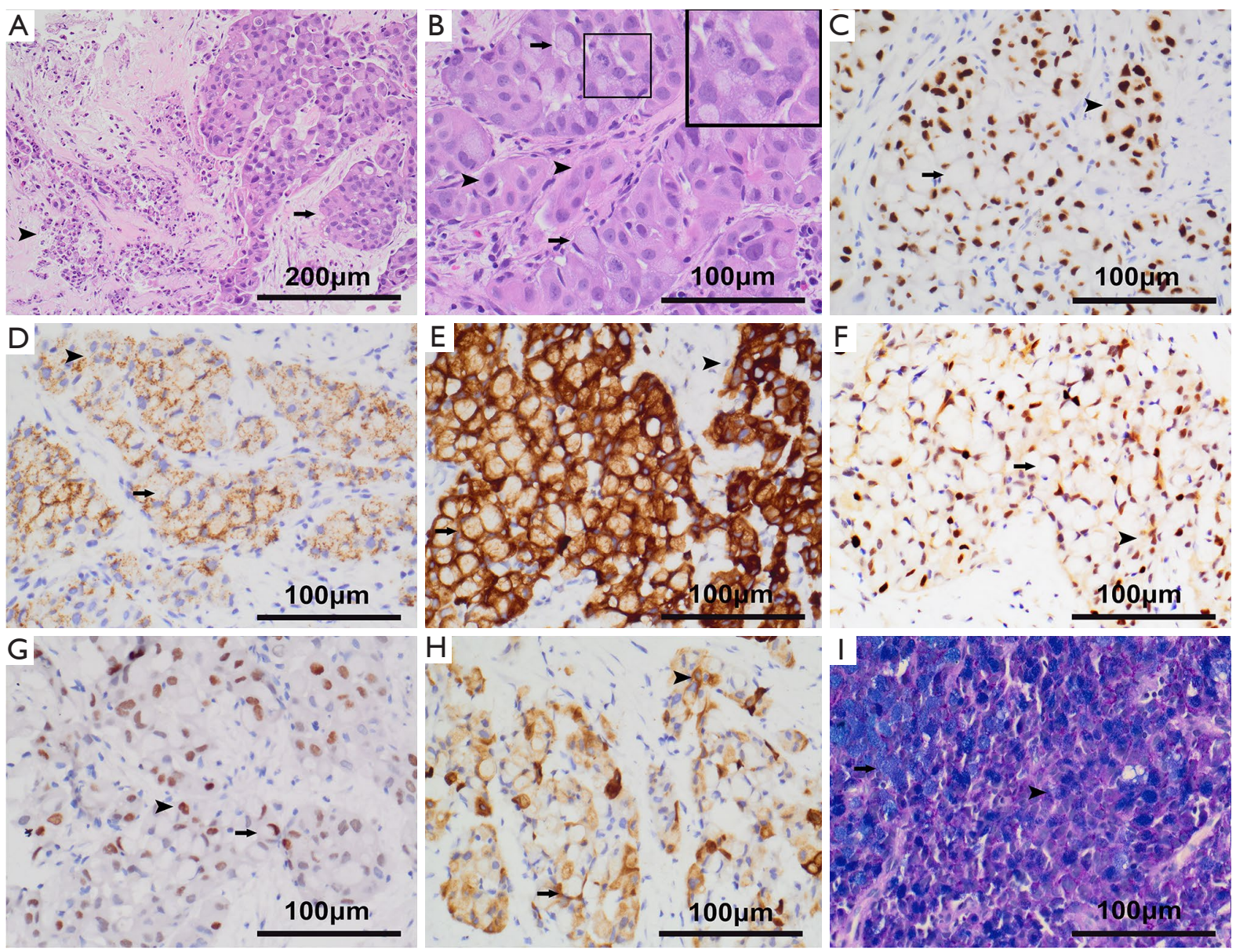

Figure 2 Hematoxylin-eosin staining (HE), immunohistochemical, and Alcian blue-periodic acid-Schiff (AB-PAS) staining of tumor cells. Histological features of biopsy show tightly arranged irregular tumor cell nests (arrow) separated by fibroconnective tissue, and necrosis could be seen (arrowhead) (HE, ×200) (A). Two morphology tumor cells could be observed: signet ring cells (arrow) and polygonal cells (arrowhead), and mitotic figures were easily found (magnification) (HE, ×400) (B). Two morphology tumor cells diffusely expressed thyroid transcription factor-1 (TTF-1) (C), Napsin A (D), CK7 (E), partially expressed P40 (F), P63 (G), and CK5/6 (H). (C-H: immunohistochemical staining, $\times 400)$. Intracytoplasmic mucin in both tumor cells was highlighted dark blue by AB-PAS staining $(\times 400)(\mathrm{I})$. [C-I: signet-ring cells (arrow) and polygonal cells (arrowhead)].

was observed (Figure 2A). The tumor was composed of two morphology cells: signet-ring cells accounted for $60 \%$ of cells, characterized by intracytoplasmic mucin and eccentrically displaced nuclei (Figure 2B), and polygonal cells accounted for $40 \%$, with fine eosinophilic granules in rich cytoplasm, round or oval nuclei, and clear nucleoli (Figure 2B). Mitotic figures were easily found (5/10 highpower fields) (Figure $2 B$ ). Immunohistochemistry revealed that the two types of cells were strongly and diffusely positive for TTF1, CK7, and Napsin A (Figure 2C,D,E); the partial expression of $\mathrm{P} 40$ (80\%), P63 (60\%), and CK5/6 (80\%) (Figure 2F,G,H); and negative for CK20 and CDX2 (not shown). Co-expression of TTF1, CK7, and Napsin $\mathrm{A}$, and $\mathrm{P} 40, \mathrm{P} 63$, and CK5/6 was easily observed (Figure $2 C, D, E, F, G, H, I$ circle labeled), and Alcian blue-periodic acid-Schiff (AB-PAS) staining showed intracytoplasmic mucin in both types of tumor cells (Figure 2I). ALK rearrangements were detected by ALK (clone D5F3) immunohistochemical assays (Figure $3 A$ ) and fluorescent in situ hybridization (FISH) (Figure 3B), and no mutation of the EGFR or KRAS gene was detected by amplificationrefractory mutation system-PCR (not shown). A final diagnosis of metastatic ALK-positive PASRF with polygonal cell morphology to the left supraclavicular 

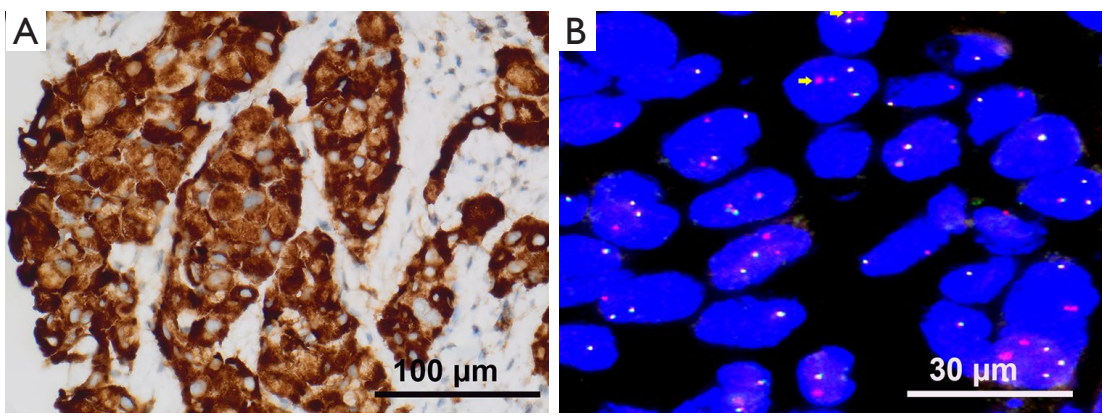

Figure 3 Detection of ALK rearrangement. Standard immunohistochemical staining for ALK(D5F3) protein of tumor cells (A). Representative ALK-rearranged tumor cells were analyzed by fluorescence in situ hybridization (FISH) using probes flanking the ALK gene (B). Yellow arrows, split FISH probes characteristic of ALK rearrangement.
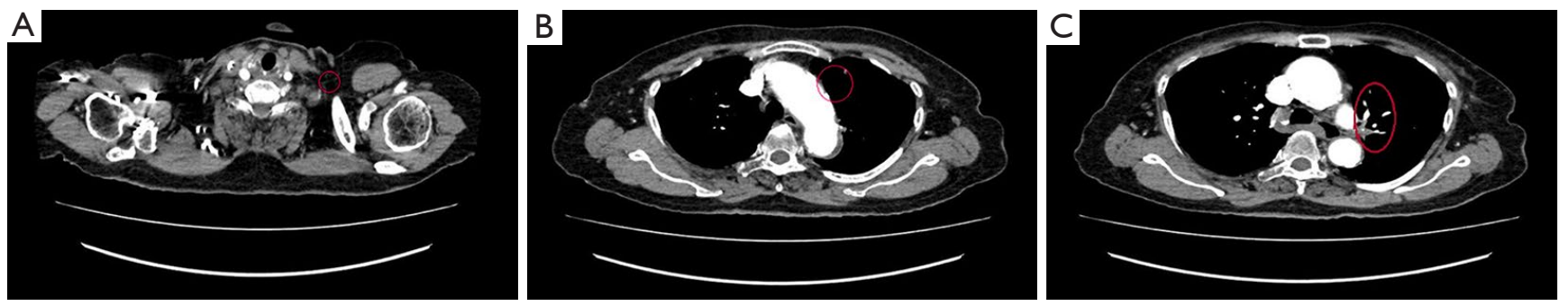

Figure 4 Computed tomography (CT) of the chest after regular treatment of Alectinib (November 25, 2020). The blue circle indicates the supraclavicular lymph node (A), mediastinal lymph nodes (B), and the lung tumor (C).

lymph node was made.

The patient was treated with Alectinib (Alecensa ${ }^{\circledR}$, Roche Pharmaceutical Ltd.), an ALK inhibitor, $600 \mathrm{mg}$ orally twice a day with meals, and the last imaging CT (November 25, 2020) (Figure 4A,B,C) showed that the size of the lung tumor $(1.6 \times 1.0 \mathrm{~cm})$ and multiple lymph nodes were much smaller than before Alectinib treatment. The serum biomarkers CEA, CA 125, and CA 15-3 all decreased to the normal range in the follow-up blood examination on November 24, 2020. The patient was still taking Alectinib regularly and was in good condition without any other special circumstances at the last follow-up on April 14, 2021.

All procedures performed in studies involving human participants were in accordance with the ethical standards of the institutional and/or national research committee(s) and with the Helsinki Declaration (as revised in 2013). Written informed consent was obtained from the patient.

\section{Discussion}

Signet ring cells are characterized by abundant intracellular mucin accumulation and a crescentic nucleus displaced toward one end of the cell. Carcinomas with signet ring cells may arise in various organs, including the stomach, colon, urinary bladder, prostate, and breast (12). Although pulmonary adenocarcinoma with signet ring features (PASRF) is relatively rare, it frequently metastasizes to lymph nodes and often harbors ALK gene rearrangements that may benefit ALK tyrosine-kinase inhibitor therapies. Here, we described a case of ALK-positive PASRF with polygonal cell morphology, which had metastasized to the supraclavicular lymph node, which demonstrates the need to diagnose PASRF correctly for therapeutic purposes.

Previous reports have indicated that a younger age of occurrence was characteristic of PASRF cases. Castro et al. (4) reported that in a series of 15 cases, ages ranged from 30 to 75 years at the time of diagnosis of PASRF with an average of 52.5 years, while in Yoshida's report (6), the mean age at presentation was 58.3 years ranging from $34-78$ years. The female patient in the present case is 85 years, which is considerably older than that seen in these previous reports.

Tsuta et al. (3) analyzed the relationship between the proportion of signet ring cell components and clinicopathologic features and revealed that compared with patients with signet ring cell components occupying $<50 \%$, patients with $\geq 50 \%$ of the lesion were younger, 
were more likely to have blood and lymph vessel invasion and lymph node metastasis, had a higher pathological stage and had a significantly lower 5-year survival rate. These findings suggest that a high proportion of signet ring cell components indicates an aggressive clinical course.

In addition to signet ring cells, which constituted $60 \%$ of the tumor in the present case, polygonal cells constituted $40 \%$, and classic adenocarcinoma was not found, which are findings not supported by other reports (5). The rarity of these cell types in primary lung tumors led us to believe metastatic spread should be considered in the differential diagnosis. When it is difficult to establish a definitive diagnosis based on histologic examination, immunohistochemical staining is often helpful, as primary pulmonary adenocarcinomas with signet ring cells frequently express TTF-1 and CK7 and are usually negative for CK20 and CDX-2. In the present case, both morphology tumor cells were diffusely immune-positive for TTF1, CK7, and Napsin A, as expected, and partially positive for P63 (70\%), as has been reported elsewhere (6). Surprisingly, a partially positive response to P40 (80\%) was observed in the two cells, and the co-expression of TTF-1, Napsin A, CK7, and P40, P63, and CK5/6 were undoubtedly present. The immunoreactivity of p63 in lung adenocarcinoma is considered uncommon, with only $5.5 \%$ of cases showing significant co-expression of TTF-1 and P63 (13), while the diffused co-expression of TTF-1 and P63 was observed in almost half of the PASRFs (6). CK5/6, P40, and P63 co-expression might suggest some degree of squamous differentiation, which would be a major pitfall during the diagnosis of PASRF. If only the metastatic polygonal cell component is present in lymph node biopsy, there is a high probability of it being misdiagnosed as squamous cell carcinoma. In the present case, the findings of diffusely positive TTF1, CK7, Napsin A, and intracytoplasmic mucin in both morphology tumor cells by AB-PAS staining supports the diagnosis of lung adenocarcinoma.

Another important differential diagnosis is mucoepidermoid carcinoma (MEC), characterized by variable components of squamoid, mucin-producing, and intermediate-type cells, with a cystic and solid growth pattern. Epidermoid cells show P63 and high molecular weight keratin expression, and $\mathrm{AB}$ staining is positive in mucin-producing cells. Most MECs are characterized by a $\mathrm{t}(11 ; 19)(\mathrm{q} 21 ; \mathrm{p} 13)$ translocation and CRTC1-MAML2 gene fusion. Based on the above differences, MECs can be ruled out in the present case.
Signet ring morphology can be observed in many other organs, including the digestive tract and the bladder, but it is very rare in the lung as a primary tumor. As a result, the diagnosis of PASRF can be made only after thorough physical examination, and a metastatic lesion from a different primary tumor is excluded. Signet ring cells commonly found in lymph nodes are often related to the digestive tract, and these tumor cells are usually CK20(+), CDX2(+), and TTF1(-) (14).

It is essential to correctly identify PASRF despite its rarity and diagnostic traps because $30-40 \%$ of PASRF are associated with ALK rearrangements and can benefit from ALK inhibitor treatment $(5,6,15)$. Signet ring cell morphology is a strong indication of ALK rearrangement (10), and more than one study has reported that ALK-positive PASRF lacks mutations of either EGFR or KRAS $(5,6)$ as our case showed. The patient in the present case received ALKtargeted therapy and has displayed a good response to treatment to date.

In conclusion, we report a case of ALK-positive PASRF with polygonal cell morphology simultaneously coexpressing TTF-1/P63/P40 metastatic to the supraclavicular lymph node. As this presentation may be misdiagnosed as other metastatic sites and histological types of tumors, it is essential to differentiate metastatic PASRF so appropriate treatment can be administered.

\section{Acknowledgments}

We would like to thank B. Draper and J. Chapnick for final English-language assistance with the manuscript.

Funding: None.

\section{Footnote}

Reporting Checklist: The authors have completed the CARE reporting checklist. Available at https://dx.doi. org/10.21037/tcr-21-335

Peer Review File: Available at https://dx.doi.org/10.21037/ tcr-21-335

Conflicts of Interest: All authors have completed the ICMJE uniform disclosure form (available at https://dx.doi. org/10.21037/tcr-21-335). The authors have no conflicts of interest to declare.

Ethical Statement: The authors are accountable for all 
aspects of the work in ensuring that questions related to the accuracy or integrity of any part of the work are appropriately investigated and resolved. All procedures performed in studies involving human participants were in accordance with the ethical standards of the institutional and/or national research committee(s) and with the Helsinki Declaration (as revised in 2013). Written informed consent was obtained from the patient for publication of this case report and accompanying images. A copy of the written consent is available for review by the editorial office of this journal.

Open Access Statement: This is an Open Access article distributed in accordance with the Creative Commons Attribution-NonCommercial-NoDerivs 4.0 International License (CC BY-NC-ND 4.0), which permits the noncommercial replication and distribution of the article with the strict proviso that no changes or edits are made and the original work is properly cited (including links to both the formal publication through the relevant DOI and the license). See: https://creativecommons.org/licenses/by-nc-nd/4.0/.

\section{References}

1. Vallonthaiel AG, Jain D, Madan K, et al. Pulmonary adenocarcinoma with signet ring features: Detailed cytomorphologic analysis. Diagn Cytopathol 2016;44:607-11.

2. Kuhn E, Morbini P, Cancellieri A, et al. Adenocarcinoma classification: patterns and prognosis. Pathologica 2018;110:5-11.

3. Tsuta K, Ishii G, Yoh K, et al. Primary lung carcinoma with signet-ring cell carcinoma components: clinicopathological analysis of 39 cases. Am J Surg Pathol 2004;28:868-74.

4. Castro CY, Moran CA, Flieder DG, et al. Primary signet ring cell adenocarcinomas of the lung: a clinicopathological study of 15 cases. Histopathology 2001;39:397-401.

5. Rodig SJ, Mino-Kenudson M, Dacic S, et al. Unique clinicopathologic features characterize ALK-rearranged lung adenocarcinoma in the western population. Clin Cancer Res 2009;15:5216-23.

Cite this article as: Tan Y, Lu X, Li Y, Liao D, Xie W, Song J, Xue L, Zhu J. ALK-positive pulmonary adenocarcinoma with signet ring features (PASRF) and polygonal cell morphology simultaneously co-expressing TTF-1/p63/P40: a case report. Transl Cancer Res 2021;10(8):3864-3869. doi: 10.21037/tcr-21-335
6. Yoshida A, Tsuta K, Watanabe S, et al. Frequent ALK rearrangement and TTF-1/p63 co-expression in lung adenocarcinoma with signet-ring cell component. Lung Cancer 2011;72:309-15.

7. Du X, Shao Y, Qin HF, et al. ALK-rearrangement in non-small-cell lung cancer (NSCLC). Thorac Cancer 2018;9:423-30.

8. Elliott J, Bai Z, Hsieh SC, et al. ALK inhibitors for nonsmall cell lung cancer: A systematic review and network meta-analysis. PLoS One 2020;15:e0229179.

9. Livieratos S, Smith JK, Fatakhov E, et al. Primary signet ring cell carcinoma of the lung: a rare subtype. BMJ Case Rep 2013;2013:bcr2013200111.

10. Boland JM, Wampfler JA, Jang JS, et al. Pulmonary adenocarcinoma with signet ring cell features: a comprehensive study from 3 distinct patient cohorts. Am J Surg Pathol 2014;38:1681-8.

11. Choi E, Lewis AL, Takei H, et al. Leptomeningeal carcinomatosis as initial presentation in adenocarcinoma of lung with signet ring cell features: an autopsy case report. Int J Clin Exp Pathol 2012;5:972-6.

12. Tsuta K, Shibuki Y, Maezawa N, et al. Cytological features of signet-ring cell carcinoma of the lung: comparison with the goblet-cell-type adenocarcinoma of the lung. Diagn Cytopathol 2009;37:159-63.

13. Ang DC, Ghaffar H, Zakowski MF, et al. Expression of Squamous Markers in Lung Adenocarcinoma (AD): Clinicopathologic and Molecular Correlates, and Implications for Differentiation from Squamous Cell Carcinoma (SqCC). Mod Pathol 2010;23:397a-a.

14. Kim JY, Kim YY, Kim SJ, et al. Predictive factors for lymph node metastasis in signet ring cell gastric cancer and the feasibility of endoscopic submucosal dissection. J Gastric Cancer 2013;13:93-7.

15. Pareja F, Crapanzano JP, Mansukhani MM, et al. Cytomorphological Features of ALK-Positive Lung Adenocarcinomas: Psammoma Bodies and Signet Ring Cells. Cancer Cytopathol 2015;123:162-70.

(English Language Editors: B. Draper and J. Chapnick) 